\title{
Custo da facoemulsificação no projeto catarata em Itápolis, SP
}

\author{
Cost of phacoemulsification in the national campaing of elective cataract \\ surgery in Itápolis, SP, Brazil
}

\author{
Roberto Saad Filho \\ Flávia Gondim Loureiro Saad ${ }^{2}$ \\ Lincoln Lemes de Freitas ${ }^{3}$
}

\begin{tabular}{l} 
RESUMO \\
\hline Objetivo: Determinar o custo médio do ato operatório da cirurgia de \\
catarata, pela técnica de facoemulsificação com implante de lente intra- \\
ocular(LIO), realizado durante a Campanha Nacional de Cirurgias Eletivas \\
de Catarata, promovido pelo Conselho Brasileiro de Oftalmologia (CBO)e \\
Ministério da Saúde na cidade de Itápolis - SP, no hospital local, entre os \\
meses de março a dezembro do ano de 2000 . Métodos: Análise de custos \\
pré e pós-operatórios a partir de coleta de dados e cálculos inserido no \\
trabalho que visam quantificar os resultados financeiros obtidos na \\
cirurgia de catarata. Para isto realizou-se estudo prospectivo de caso em \\
série. Um grupo com catarata senil submeteu-se à intervenção cirúrgica. \\
Foram verificados neste estudo 58 cirurgias de catarata. Resultados: O \\
custo médio do ato operatório foi de R\$ 485,03 ou US\$248,05. Este valor \\
representa o custo médio da intervenção cirúrgica propriamente dita; em \\
que determinadas insumos, equipamentos, taxas e/ou outros serviços \\
foram previamente determinados e utilizados para este projeto. Conclusão: \\
A técnica de facoemulsificação na cirurgia de catarata, tem-se tornado \\
procedimento cada vez mais utilizado devido à sua comprovada eficácia, \\
sendo método de excelência no tratamento da catarata. Apesar desta \\
técnica encontrar-se solidificada, deparamo-nos com um problema de \\
ordem econômica, pois o seu custo ainda a faz inacessível a uma parcela \\
da população.
\end{tabular}

Descritores: Facoemulsificação/economia; Custos e análise de custo; Extração de catarata/economia; Custos diretos de serviços; Promoção da saúde

\footnotetext{
${ }^{1}$ Mestre profissional da prática oftalmológica - MBA Mestrado Profissionalizante - Universidade Federal de São Paulo - UNIFESP. Médico oftalmologista do Hospital - Santa Casa de Misericórdia e Maternidade Dona Julieta Lyra - Itápolis.

${ }^{2}$ Médica oftalmologista do Hospital - Santa Casa de Misericórdia e Maternidade Dona Julieta Lyra - Itápolis. ${ }^{3}$ Doutor em Oftalmologia pela Universidade Federal de São Paulo - UNIFESP, colaborador do Setor de Catarata do Departamento de Oftalmologia da Universidade Federal de São Paulo - UNIFESP.

Endereço para correspondência: Roberto Saad Filho. Av. Frei Paulo Luig, 298 - Itápolis (SP) CEP 14900-000 E-mail: versaad@aol.com

Recebido para publicação em 31.05.2004

Versão revisada recebida em 04.10.2004

Aprovação em 30.08.2004
}

\begin{tabular}{l}
\hline INTRODUÇÃO \\
\hline Considerando que a catarata seja a principal, ou uma das principais \\
causas de cegueira no curso da humanidade. Atualmente, estima-se que, no \\
mundo, existam 25 milhões de pessoas cegas com acuidade visual menor \\
que 20/400 bilateral vítimas de catarata. Avaliam-se que haja 110 milhões de \\
olhos com catarata com uma acuidade visual menor que $20 / 200^{(1)}$. No Brasil, \\
a estimativa é de 4 milhões de pessoas portadoras de alguma deficiência \\
visual e 1,2 milhões de cegos. Acredita-se que anualmente surgem no país \\
120 mil novos casos de catarata \\
Entre os fatores de riscos para o desenvolvimento da catarata destacam- \\
se: doença pré-existentes (por exemplo: diabete melito e glaucoma), uso de \\
determinadas drogas (álcool, fumo, esteróides e outras), exposição à radia- \\
ção ultravioleta, desidratação, desnutrição, fatores demográficos (morado- \\
res de área rural, baixo nível educacional, baixo peso, baixa estatura e
\end{tabular}


outros). Porém, o maior fator de risco para a opacificação do cristalino é o envelhecimento ${ }^{(1)}$.

A longevidade e a necessidade de comunicação visual trazem uma influência direta no relacionamento do ser humano dentro da sua comunidade, pois a necessidade de independência e participação socioeconômica está também diretamente relacionada com a saúde ocular. A cegueira pela catarata além de incapacitar o indivíduo levando a uma limitação da capacidade de trabalho, representa um elevado ônus para a sociedade e ao país. Diferentemente da maioria das condições degenerativas e incapacitantes, a cegueira por catarata tem cura na maioria dos casos e esta restauração só pode ser conseguida com o tratamento cirúrgico ${ }^{(3-9)}$.

A partir de estudos epidemiológicos em 1999 o Ministério da Saúde em parceria com o Conselho Brasileiro de Oftalmologia, implementou a $1^{\underline{a}}$ Etapa da Campanha Nacional de Cirurgia Eletiva Mutirão da Catarata. Esta campanha teve como objetivo aumentar a oferta de cirurgias de catarata. No ano de 2000 , esta iniciativa foi realizada em 636 municípios da Federação ${ }^{(2)}$. Entre os favorecidos destaca-se o município de Itápolis, localizado no interior do estado de São Paulo, onde no único e filantrópico hospital da localidade foi realizado este trabalho.

A restauração da visão pela cirurgia da catarata produz amplos benefícios econômicos e sociais; um estudo da Índia rural demonstrou que indivíduos, após a cirurgia de catarata, aumentaram sua produtividade anual em cerca de $1.500 \%$ do valor do custo da cirurgia ${ }^{(10)}$.

As técnicas operatórias em vigência mais usadas para a catarata são: facectomia extracapsular com implante de lente intra-ocular e facoemulsificação com implante intra-ocular. Atualmente e categoricamente a facoemulsificação é a que proporciona a reabilitação visual mais precoce, sendo consagrada como a técnica de excelência para o tratamento da catarata; uma grave desvantagem desta técnica cirúrgica é o seu custo, quer seja na curva do aprendizado contínuo, bem como no custo da aquisição e manutenção dos equipamentos e de determinados insumos ${ }^{(11-12)}$.

O objetivo deste estudo é apresentar o custo médio da cirurgia de facoemulsificação "no ato operatório em si" e se possível estender uma discussão, por uma medicina mais digna, ética e justa com escasso recurso financeiro.

\section{MÉTODOS}

Realizou-se um estudo de caso em série prospectivo, num grupo de 58 pacientes com catarata senil, que foram submetidos à facoemulsificação com implante de LIO. Os pacientes foram selecionados no Serviço Municipal de Saúde, no período de março a dezembro de 2000.

No critério de inclusão os pacientes selecionáveis para este estudo, deveriam preencher os seguintes quesitos: portadores de catarata senil; idades iguais ou maior que 50 anos; acuidade visual igual ou inferior a 0,4 com estenopéico em um, ou ambos os olhos; submeter-se a um teste semiológico oftalmológico que consistia do seguintes exames: anamnese, ectoscopia, refração, biomicroscopia, fundoscopia, tonometria, ceratometria e biometria ultra-sônica; avaliação clínica pré-anestésica, sendo realizado os exames complementares: hemograma completo, glicemia jejum, coagulograma I, creatinina e eletrocardiograma. Paciente e responsável assistiam a um vídeo explicativo desenvolvido pelo Instituto da Catarata da UNIFESP e recebiam panfletos explicativos sobre os cuidados pré, intra e pós-operatório.

Os critérios de exclusão, eram aplicados aos pacientes que não preenchiam a todos os quesitos de inclusão.

Todas as cirurgias foram realizadas, por único cirurgião, sob anestesia local com a técnica peribulbar e sedação. As mesmas eram realizadas em caráter ambulatorial onde os pacientes recebiam alta hospitalar imediata ao ato operatório. Procurou-se agendar no mínimo 2 cirurgias por período.

Aos oftalmologistas envolvidos neste estudo, ficaram a obrigação de fornecer e realizar a manutenção dos seguintes equipamentos: um microscópio cirúrgico Zeiss modelo OPMI; um aparelho de facoemulsificador Allergan modelo diplomax; instrumentais cirúrgicos (pinças, lâminas de safira e etc.); e um consultório oftalmológico completo para o controle pré e pós- operatório.

Ao hospital era designado o fornecimento e gerenciamento dos itens: locação e manutenção do prédio com seus custos diretos e indiretos; serviço do centro cirúrgico e esterilização, suporte nos equipamentos destinados ao anestesista; e recebimento, rateio e gerenciamento do montante em espécie.

A obtenção dos dados para análise dos custos deste trabalho foram colhidos do relatório analítico do sistema de informática do Hospital - Santa Casa de Misericórdia de Itápolis. Os critérios de levantamento dos preços para a determinação desses custos, são obtidos basicamente de duas formas: primeiramente a Instituição fornece tais importâncias: taxa de sala, serviços profissionais e os custos dos matérias e insumos e por fim, os valores da utilização dos equipamentos foi determinada pelo autor onde se utilizou a lógica do "retorno do investimento", metodologia aplicada e proferida durante o curso de Mestrado Profissionalizante da UNIFESP. O projeto deste estudo foi previamente aprovado pelo comitê de Ética em Pesquisa da UNIFESP.

\section{RESULTADOS}

O custo médio do ato operatório, foi de $\mathrm{R} \$ 485,03$ ou US\$ 242.72 (a conversão da moeda brasileira real para o dólar comercial americano foi na data de 31/12/2000). Este valor representa o custo médio da intervenção cirúrgica propriamente dita; em que determinados insumos, equipamentos, taxas e ou outros serviços foram previamente determinadas e utilizados para este projeto. Cabe salientar que a escolha da técnica e instrumentos fora escolha pessoal do cirurgião, em relação aos insumos e as medicações usadas ficaram a cargo da conveniência do hospital.

Os resultados obtidos das 58 cirurgias (Tabela 1), na coluna Histórico é feita uma descrição e especificação dos itens 


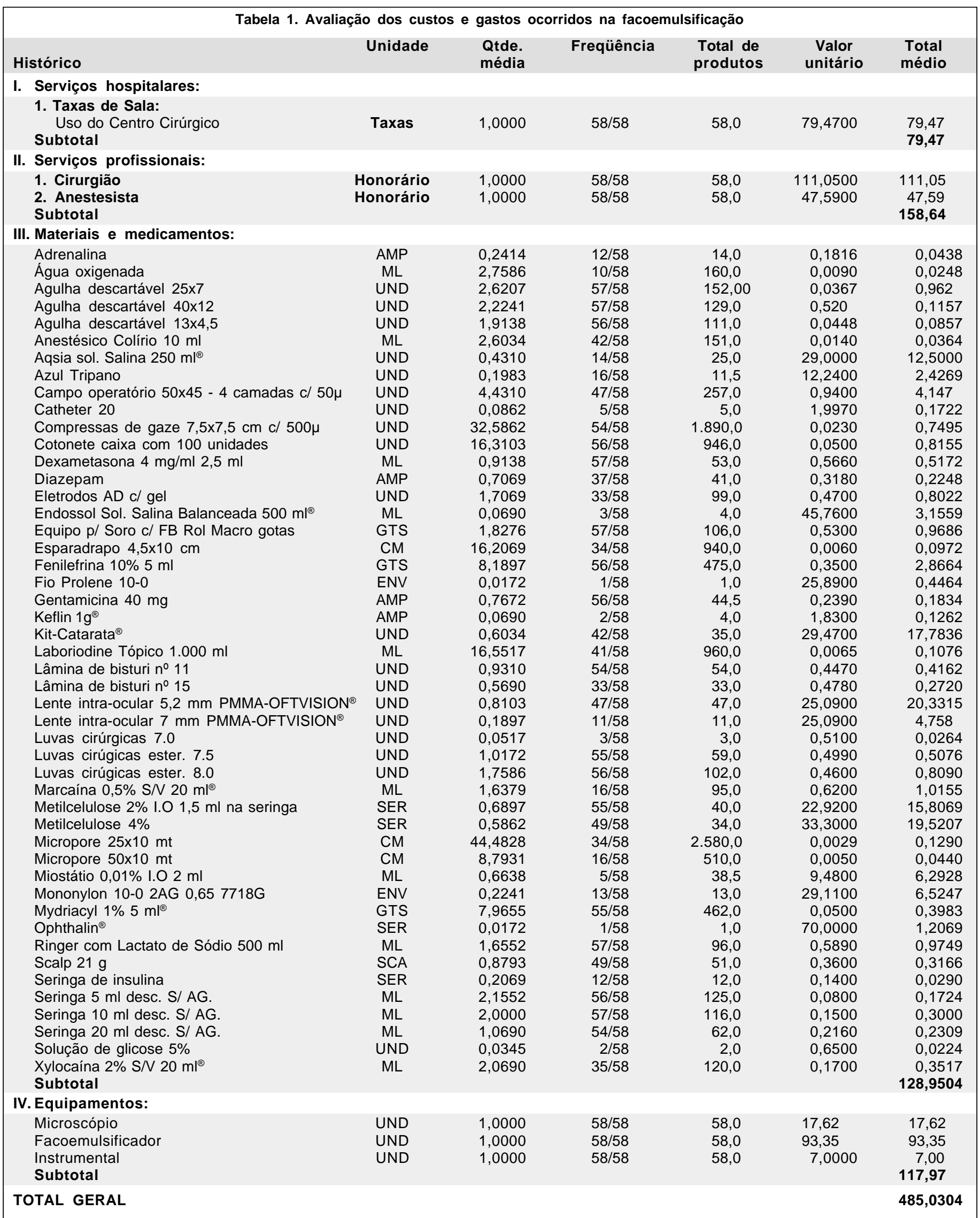


utilizados no ato operatório. Estes foram; serviços hospitalares e profissionais, material, medicamento e equipamento são analisados na sua apresentação (unidade - aspecto), quantidade média (é o número total de vezes utilizado dividido pela frequiência), freqüência (é o número surgido em cada caso dividido pelo número total de casos), totalidade (é a quantidade total de vezes em que o item surgiu), valor unitário (preço médio de custo dos produtos adquiridos no ano de 2000), e seu respectivo valor médio (é o valor unitário multiplicado pela quantidade média). A partir daí, fez-se uma somatória dos valores de cada item, em que se obtêm o total geral ou custo médio da cada ato operatório.

Para determinar o preço de uso dos equipamentos por cirurgia, utilizou-se a lógica do retorno do investimento, sendo esta metodologia assim equacionada: o valor total da aquisição do equipamento foi dividido pelo valor da depreciação vida útil (em meses), neste montante soma-se o custo da manutenção ( $1 \%$ do valor da aquisição), mais custo anual do seguro do equipamento, este valor é então dividido pelo número de vezes de utilização do equipamento ao mês.

É importante justificar a existência de algumas variantes: a determinação do tempo de depreciação dos equipamentos partiu de um consenso entre o autor e o orientador deste estudo; o valor da taxa de sala foi determinado por "critérios" definidos pela direção do hospital e não tivemos acesso a tal; o valor dos serviços médicos para este trabalho foi definida numa reunião prévia entre médicos e direção hospitalar; também não se computou os valores referentes a: custos com exames complementares clínicos e oftalmológicos pré-operatório, gastos com atendimento ambulatorial pré e pós-operatório e finalmente não se calculou os impostos e/ou qualquer variável do sistema financeiro como a inflação deste período. Salienta-se que neste trabalho não se importou em fazer uma análise dos resultados clínicos pós-operatório.

\section{DISCUSSÃO}

A literatura tem sido consensual na importância da cirurgia de catarata pelo método de facoemulsificação principalmente pelos resultados clínicos pós-operatório obtidos ${ }^{(1,11-12)}$. Apesar desta técnica encontrar-se solidificada, deparamo-nos com um problema de ordem econômica, pois seu custo ainda a faz inacessível a uma parcela da população ${ }^{(11,13)}$.

A preocupação com custos é algo que mobiliza as consciências dos países desenvolvidos, e deveria principalmente nortear aqueles em desenvolvimento, onde é notório as fragilidades: políticas, econômicas e sociais; para tanto seria mais coerente e factível, uma ampla e massiva discussão sobre meios ou formas de se diminuir os custos sem afetar a qualidade, fazendo-se que o melhor da medicina possa ser oferecido a população.

Segundo dados da literatura, em 1987 relatou que o custo de uma cirurgia de catarata nos Estados Unidos da América pode variar de US $\$ 3,000$, a US $\$ 6,000$, porém o mesmo autor relata que dependendo da tecnologia empregada uma cirurgia de catarata pode apresentar um custo muito baixo variando de US\$ 5, a US\$ 20 $0^{(14)}$. Alguns autores, em 1994 descreveram o custo da facectomia extra-capsular realizado no Hospital das Clínicas da Unicamp foi de US\$77,00 ${ }^{(15)}$. Outro artigo apresentado m 1998, descreve que em um hospital na Espanha o custo com materiais para a cirurgia de facoemulfisicação foi de 284,94 euros e na facectomia extra-capsular foi 131,22 euros, porém, quando é feito uma análise excluindo os gastos com os materiais e computando-se apenas os gastos com o tempo de centro cirúrgico e ambulatório, a facoemulsificação torna-se uma cirurgia mais barata ${ }^{(16)}$.

Este estudo evidenciou-se que o custo médio por cirurgia pela técnica de facoemulsificação foi de R $\$ 485,03$ ou US\$ 248,05 . Destaca-se que a imensa maioria dos materiais e medicamentos utilizados são de origem nacional, em especial citamos a lente intra-ocular e os viscoelásticos, estes itens por si só podem fazer muita diferença na contabilidade final. Ainda sobre a lente intra-ocular seria interessante avaliar a utilização das mesmas dobráveis ou expansíveis, pois poderíamos eliminar o fio de sutura, podendo proporcionar uma melhor recuperação pós-operatória, além da possibilidade de uma menor incidência de opacidade capsular, o que diminuiríamos um gasto futuro com a capsulotomia e seus possíveis riscos inerentes.

\section{CONCLUSÃO}

Citamos a necessidade de estudos consistentes sobre o tema, custo-benefício ou efetividade-qualidade, dos equipamentos, produtos e/ou insumos, nacionais comparando-os entre si, e/ou confrontá-los com os de origem importadas, já que o preço são significativamente diferentes. Outro quesito se refere a utilização de produtos recicláveis, reesterelizáveis como forma de diminuição de custo; também não podendo deixar de citar o cuidado com o desperdício ou uso abusivo e dispendioso de certas substâncias "descartáveis".

Apesar deste trabalho não ter elaborado um protocolo onde poderia sugerir a quantidade padrão de serviços, materiais e equipamentos, para a cirurgia de catarata, é importante salientar que se deve empreender uma maior reflexão sobre este tema, pois ao se introduzir um protocolo, o mesmo servirá como um importante fator de controle de custo.

É interessante acrescentar que este trabalho seria ainda mais interessante, caso apresentasse um estudo comparativo e se necessário com cálculos específicos, onde compararíamos os valores pagos pelo sistema público de saúde e os diversos convênio vigentes no mercado.

É louvável discorrer sobre alguns assuntos como: a realização em ambiente ambulatorial comparado ao hospitalar, curva de aprendizado do cirurgião, custos decorrentes das complicações operatórias, etc. Outros trabalhos deverão ser realizados no futuro como forma de dar continuidade a esta investigação. 


\section{ABSTRACT}

Purpose: To determine the average cost of cataract surgery using the phacoemulsification technique with intraocular lens implant (IOL). This study was conducted during a national campaign to treat patients with cataracts promoted by the Brazilian Council of Ophthalmology (CBO) and the Health Ministry in the city of Itápolis - SP from March to December, 2000. Methods: All expenses related to the surgery were collected (pre-, intra-, and postoperative data) and analyzed. Fifty-eight patients with senile cataracts and without any other ocular findings were submitted to the procedure in a prospective study. Results: The average cost of the surgery in this study was $\mathrm{R} \$ 485.03$ or US\$248.05. This value represents the average cost of the surgery itself; in which some materials, equipment, taxes and other services were previously determined and used for this project. Conclusion: The phacoemulsification technique for cataract surgery has been used more and more, due to its effectiveness, being an excellent method to treat cataracts. Although this is an excellent technique, the economical aspect does not allow it to be used for part of the population

Keywords: Phacoemulsification/economics; Costs and cost analysis; Cataract extraction/economics; Direct service costs; Health promotion

\section{REFERÊNCIAS}

1. Apple DJ, Ram J, Foster A, Peng Q. Elimination of cataract blindness: a global perspective entering the new millennium. Surv Ophthalmol. 2000;45(Suppl 1):S1-196.
2. Conselho Brasileiro de Oftalmologia. Relatório 2000. Campanha nacional de cirurgia eletiva de catarata e campanha de Redução da cegueira decorrente da retinopatia diabética. São Paulo: Conselho Brasileiro de Oftalmologia, Ministério da Saúde; 2000.

3. Drummond MF. Economic aspects of cataract. Ophthalmology. 1988;95(8): 1147-53.

4. O'Day DM. Management of cataract in adults. Am Fam Physician. 1993; 47(6):1421-30.

5. Weinstock FJ. The cataract revolution: What the primary care physician needs to know. Geriatrics. 1993;48(6):52-6.

6. Kara José JR N, Schellini SA, Silva MRBM, Bruni LF, Almeida AGC. Projeto catarata - Qual a sua importância para a comunidade?. Arq Bras Oftalmol. 1996;59(5):490-3.

7. Prado JR J, Silva ALB, Alves MR, Kara José N, Temporini ER. Tratamento cirúrgico da catarata senil no Hospital das Clínicas da Faculdade de Medicina da Universidade de São Paulo. Aspectos da rotina operacional. Rev Méd (São Paulo). 1997;76(3):192-6.

8. Kara José N, Temporini ER. Cirurgia de catarata: o porquê dos excluídos. Rev Panam Salud Publica. 1999;6(4):242-7.

9. Belfort JR R. Editorial; honorários médicos, sistema único de saúde e ensino da oftalmologia para o século XXI. Arq Bras Oftalmol. 1999;62(6):675-6.

10. Kara-José N, organizador. Prevenção da cegueira por catarata. Campinas: UNICAMP; 1996.

11. Saad Filho R. Custo da cirurgia no projeto catarata em Itápolis [tese]. São Paulo: Universidade Federal de São Paulo - Escola Paulista de Medicina; 2002.

12. Freitas LL, Di Martino DS, Mori E, Mendonça M, Casanova FHC, Abreu MT. Estudo prospectivo comparativo de duas técnicas cirúrgicas de extração extracapsular planejada de catarata com implante de lente intra-ocular: incisão limbar e incisão escleral tunelizada. Arq Bras Oftalmol. 2001;64(3):239-46.

13. Ferraz EVAP, Lima CA, Cella W, Arieta CEL. Adaptação de questionário de avaliação da qualidade de vida para aplicação em portadores de catarata. Arq Bras Oftalmol. 2002;65(3):293-8.

14. Schwab L. Cost-effective cataract surgery in developing nations. Ophthalmic Surg. 1987;18(4):307-9.

15. Kara José N, Delgado AMN, Arieta CEL. Exeqüibilidade da cirurgia de catarata em hospital-escola: em busca de um modelo econômico. Rev Assoc Méd Bras. 1994;40(3):186-8.

16. Castells X, Comas M, Castilla M, Cots F, Alarcón S. Clinical outcomes and costs of cataract surgery performed by planned ECCE and phacoemulsification. Int Ophthalmol. 1998;22(6):363-7.

\title{
XXX Congresso da Sociedade Brasileira de Retina e Vítreo
}

\section{4 a 16 de abril de 2005 Hotel Blue Tree Angra dos Reis - RJ}

\section{INFORMAÇÕES:}

\author{
LK Assessoria e Promoções \\ Tel.: (21) 3878-2512/ 3878-1610 \\ Fax: (21) 3878-1548 \\ E-mail: evento@ Ik.com.br \\ Home-page: www.retina2005.com.br
}

